\title{
A Theory on Predatory Advertising After A Demand-reducing Shock*
}

\author{
Yutian Chen $^{\dagger} \quad$ Wei Tan ${ }^{\ddagger}$
}

October 8, 2009

\begin{abstract}
We model the advertising competition between an incumbent and a new entrant, after the new entrant suffers a demand-reducing shock. The incumbent is privately informed as to the effect of the shock, and has incentive to signal the entrant its private information in order to induce the entrant's exit. Our fundamental result is that, there exist separating equilibrium and pooling equilibrium after the refinement by intuitive criterion. In both of them, the incumbent's advertising is distorted upward as a consequence of signaling. Moreover, such distortion occurs only among the markets where loyalty to the entrant falls in the intermediate range. In the rest of the markets, the incumbent advertises at its normal competitive level in the absence of predation. The theoretical prediction offers a rationale to the empirical findings in Chen and Tan (2009).
\end{abstract}

Keywords: Predatory advertising, Exit, Incomplete information

JEL Classification: D43, L11, L13

\footnotetext{
* We would like to thank Hugo Benitez-Silva, Sandro Brusco, Pradeep Dubey, Sara Ellison, Ginger Jin, Qihong Liu, Konstantinos Serfes, Matthew Shum, Yair Tauman for helpful discussions and comments. We also appreciate the suggestions from seminar participants at Queens College, Drexel University, 2007 International Industrial Organization Society meetings and 2007 Econometric Society Summer meetings.

${ }^{\dagger}$ Department of Economics, California State University, Long Beach, CA 90840, USA. Email: ychen7@csulb. edu

${ }^{\ddagger}$ Department of Economics, SUNY-Stony Brook, NY 11794, USA. Email: wtan@ notes. cc. sunysb. edu
} 


\section{Introduction}

The theoretical literature identifies several ways through which advertising is strategically used to signal private information. For example, advertising signals the unobserved product quality of experience good (Milgrom and Roberts (1986)); advertising signals cost/demand by the incumbent as a means of deterring entry (Bagwell and Ramey (1988, 1990)); and advertising signals unobserved demand by manufacturer to retailers (Albæk and Overgaard (1992)). However, few existing studies explore advertising as a signal in predatory practice.

The basic idea of predation is that, a firm may sacrifice part of the profits that could be earned under competitive circumstances in order to induce the exit of an opponent, therefore gaining additional profits in the future. In this work, we develop a theoretical model where advertising signals unobserved market demand in order to induce the exit of an opponent.

We model the competition via demand-enhancing advertising between an incumbent and a new entrant in multiple independent markets after the entrant suffers a demandreducing shock. The incumbent knows the impact of the shock on market demand in virtue of its experience, whereas the new entrant does not know. Consider a two-period game in a single market. In period one, nature chooses the effect of the shock as either severe or mild, which is only observed by the incumbent. The incumbent and the entrant then choose advertising level simultaneously, and each has its demand/profit realized. In period two, the entrant makes its withdrawal decision based on its period-one demand. If it exits, the incumbent becomes a monopolist, otherwise the effect of the shock is revealed to the entrant and they compete as duopolists.

An important feature of our model is, we allow for the heterogeneity in market's loyalty towards the entrant prior to the news event. Predation is either impossible or unnecessary in markets where loyalty to the entrant is either too strong or too weak. This 
gives the incumbent different incentives to predate across markets. More specifically, following the bad news, the demand in markets which a priori had a weak loyalty to the entrant is driven down to such a low level, that the entrant will choose to exit even if the shock has only a mild impact. Similarly, in those markets which a priori had a strong loyalty to the entrant, the entrant will always stay in spite of the effect of the shock. Consequently, the incumbent's predatory intent can only exist in those markets where loyalty to the entrant falls in the median range.

We find that, in markets with median loyalty to the entrant, there exist two types of equilibrium after invoking intuitive criterion (Cho and Kreps (1987)) to refine the equilibrium set: separating equilibrium and pooling equilibrium. First, there is a unique intuitive separating equilibrium, in which the incumbent advertises according to its one-period optimal when the shock is mild, but upward-distorts its advertising when the shock is severe to signal the entrant an intrinsically unprofitable market circumstance. Second, there exist a continuum intuitive pooling equilibria. In the least cost one, the incumbent advertises according to its one-period optimal when the shock is severe, but upward-distorts its advertising when the shock is mild to disenable the entrant's reference of the real effect of the shock. The fundamental distortion occurring in both separating and pooling equilibrium is an advertising-enhancing distortion.

Our work is motivated by the empirical study of Chen and Tan (2009), where they examine the change in advertising behavior of incumbent firms after a new entrant encounters a bad news and might exit. Their work is based on a well known case in the pharmaceutical industry. Crestor, a brand that belongs to a therapeutic class of Statin drugs, first entered the market in August 2003. Shortly after its entry, several cases of severe side effects occurred from the use of this brand. On March 4, 2004, Public Citizen, a very influential consumer advocacy group, submitted a petition to the Food and Drug Administration (FDA) to formally remove the brand from the market. Major news 
network immediately broadcasted the news about the petition from Public Citizen.

The central question in Chen and Tan (2009) is to investigate if after the news, Crestor's competitors strategically advertised ${ }^{1}$ in order to accelerate the withdrawal of Crestor. As the bad news did not generate the pressure for Crestor to fully withdraw from the Statin drug market, the exit decision is more relevant at the physician level. Chen and Tan (2009) treat each physician as a market, and consider Crestor as having exited from a physician market if it foregoes relationship building with that physician by ceasing to detail (i.e., send out pharmaceutical sales representatives to visit at physicians' offices) the physician. Their major finding is, in the period immediately following the bad news of Crestor, the dominant brand of Statin drugs, Lipitor, demonstrates a significantly larger increase in its advertising towards physicians with whom Crestor has a median share in physician prescriptions, relative to the increase in its advertising among physicians with either a low or high Crestor share.

While it is not straightforward how such advertising pattern can be justified, the theoretical prediction in our work, based on asymmetric information assumption, offers a possible explanation. We show that, the empirical findings in Chen and Tan (2009) can be the result of Lipitor's predation over Crestor after the bad news event.

Consider each physician as a market, and Crestor's share in physician's total prescriptions prior to the shock captures the market loyalty to Crestor. When Lipitor is privately informed with the severity level of the shock, among physicians who have a median loyalty to Crestor, Lipitor biases its advertising upward with the purpose of either convincing Crestor an inherently unprofitable market due to a severe shock (separating equilibrium), or disenabling Crestor to infer the mild effect of the shock (pooling equilibrium). In either of these equilibria, Lipitor is inducing Crestor to exit by advertising more than its

\footnotetext{
${ }^{1}$ Pharmaceutical firms mainly compete through advertising and marketing promotions. The major form of advertising is detailing, referring to visit of pharmaceutical sales representatives at physicians' offices.
} 
one-period optimal. Whereas, among the rest of the physicians, Lipitor advertises at its one-period optimal level since signaling is either unnecessary or unprofitable. As such, Lipitor's predatory incentive leads to an upward distortion in its advertising only among physicians with a median loyalty to Crestor.

Our work mainly makes two contributions. First, to our knowledge, this is the first work which formally models advertising as a signal of unknown demand in order to drive out a competitor. Second, the lack of empirical evidence on predatory practice is mainly due to the difficulty in differentiating a predatory behavior from a normal competitive behavior. Our model examines the link between predation and market's property, thus offers empirically testable prediction on predation.

The rest of the paper is organized as follows: Section 2 reviews the literature; Section 3 introduces the background regarding the Statin drug market and the empirical findings in Chen and Tan (2009); Section 4 is the theoretical model and main results; Section 5 relates the theoretical prediction to the empirical results; and Section 6 concludes.

\section{Literature Review}

Our work is closely related to the theory on predation. Beginning in the 1950s, "Chicago School" economic analysis, assuming complete information, rightfully pointed out the irrationality of monopolizing a market through predation $(\mathrm{McGee}(1958,1980))$. On the other hand, modern strategic analysis have resurrected the logic of predatory practice. The key assumption is the absence of perfect information; under such conditions, predation becomes a rational strategy, even for a predator without efficiency or financial advantages over its prey. Many intrinsic incentives have been identified in favor of predation. For example, Milgrom and Roberts (1982) and Kreps and Wilson (1982) show that "reputation effect" justifies predation as an equilibrium, since preying against a rival fosters a "tough" 
reputation that discourages future challenges. Fudenberg and Tirole (1986) use "signal jamming" to illustrate that when a new entrant needs to infer its future profitability from its current profit, a predator is able to interfere with the entrant's inference procedure as a means of encouraging its exit. Roberts (1986) shows that preying entails an upward distortion in the predator's quantity, in order to signal a low production cost to the entrant therefore to deter its entry. Saloner (1987) depicts predation in mergers, where the acquiring firm may expand output to signal a low cost for the purpose of achieving better takeover terms.

The theoretical literature identifies several strategic usages of advertising to deter entry. Based on the "goodwill" effect of advertising where advertising generates "captive" consumers, Schmalensee (1983), Fudenberg and Tirole (1984) show that by underinvesting in advertising, an incumbent is able to commit on a fierce post-entry competition therefore can deter entry. Instead, with a dynamic setting, Doraszelski and Markovich (2007) find that an incumbent may deter entry by overinvesting in advertising. Another line of rationale is based on the "signaling" effect of advertising. Bagwell and Ramey (1988, 1990), Linnermer (1998) argue that, an incumbent may distort its advertising to signal its private information regarding cost/demand/quality, aimed at deterring entry.

Few literature exists on advertising in predatory practice aimed at inducing rivals to exit. Hilke and Nelson (1984) informally argue that a new entrant is more vulnerable to an incumbent's advertising at its initial period of market penetration, therefore an incumbent may overinvest in advertising to raise the entrant's advertising cost, in order to force it to exit. Built upon the "noisy advertising" argument in Hilke and Nelson (1984), Mason (1986) models the advertising competition between an incumbent and a recent entrant where consumers are imperfectly informed of products' attributes. He finds that, the incumbent may distort its advertising to misrepresent its product's attribute, in order to reduce the apparent novelty of the entrant's product and lead to the entrant's exit. 


\section{Background and Empirical Findings}

Our work is motivated by a case in the Statin drug market. Statins are drugs that help lower cholesterol levels. With at least 12 million Americans taking cholesterol-lowering drugs - most of them statins - and experts recommending that another 23 million take them, Statin drugs are certainly very important to the pharmaceutical industry. With sales for 2004 reaching US $\$ 10.8$ billion and 5.2 billion respectively, the top two brands in the market, Lipitor and Zocor, are not only the largest seller for their maker, Pfizer and Merck $\&$ Co, but also the largest two selling drugs in the world.

The four main brands in the Statin drug market are Lipitor, Zocor, Pravachol and Crestor. Their makers are Pfizer, Merck, Bristol-Myers Squibb and Astra Zeneca respectively. Among them, Lipitor and Crestor are considered closer substitutes as they use the same production method (Synthetic). Moreover, Lipitor is the dominant seller accounting for more than $40 \%$ market share.

Crestor first obtained FDA approval on August 12th, 2003, and entered the market shortly afterward. After entering the market, several severe cases of side effects occurred. Alarmed by the findings, a very influential consumer advocacy group, known as Public Citizen, submitted on March 4th, 2004, a formal petition to the FDA to have the drug removed from the market. Major news network, such as CBS, immediately broadcasted the news about the petition from Public Citizen. Crestor's maker AstraZeneca wouldn't comment on any of the serious side effects, except to say that "the safety profile is totally comparable" to what pre-marketing studies had predicted.

Interestingly, this is not the first time that Public Citizen issued a petition for the removal of a Statin drug. Baycol, another Statin drug, withdrew from the market in the fall of 2001 following a petition by Public Citizen to the FDA. The side effects of Baycol were very similar to those of Crestor, in that they were both likely to cause kidney damage.

Chen and Tan (2009) argue that this case provides a unique opportunity to test the 
theory on predation. The unanticipated news event seriously lowered Crestor's demand, but is not severe enough to cause Crestor to pull out immediately. While Crestor remains active in detailing, its competitors may have incentive to employ predatory strategy to convince Crestor that staying is no longer profitable. Empirical evidence in Chen and Tan (2009) shows that, after the bad news, Lipitor demonstrates a significantly larger increase in its advertising among physicians with whom Crestor has a median market share, relative to the change in its advertising among physicians where Crestor's market share is either low or high. These results are robust under various empirical model specifications.

Figure 1 in Chen and Tan (2009) plots the number of Lipitor's advertising before and after the news event and the quantile of Crestor's market share. As the figure shows, when Crestor's market share is between the 40th and 70th quantile, Lipitor exhibits the largest increase in its advertising after the news event. As such, the change in Lipitor advertising is non-monotonic in Crestor's market share.

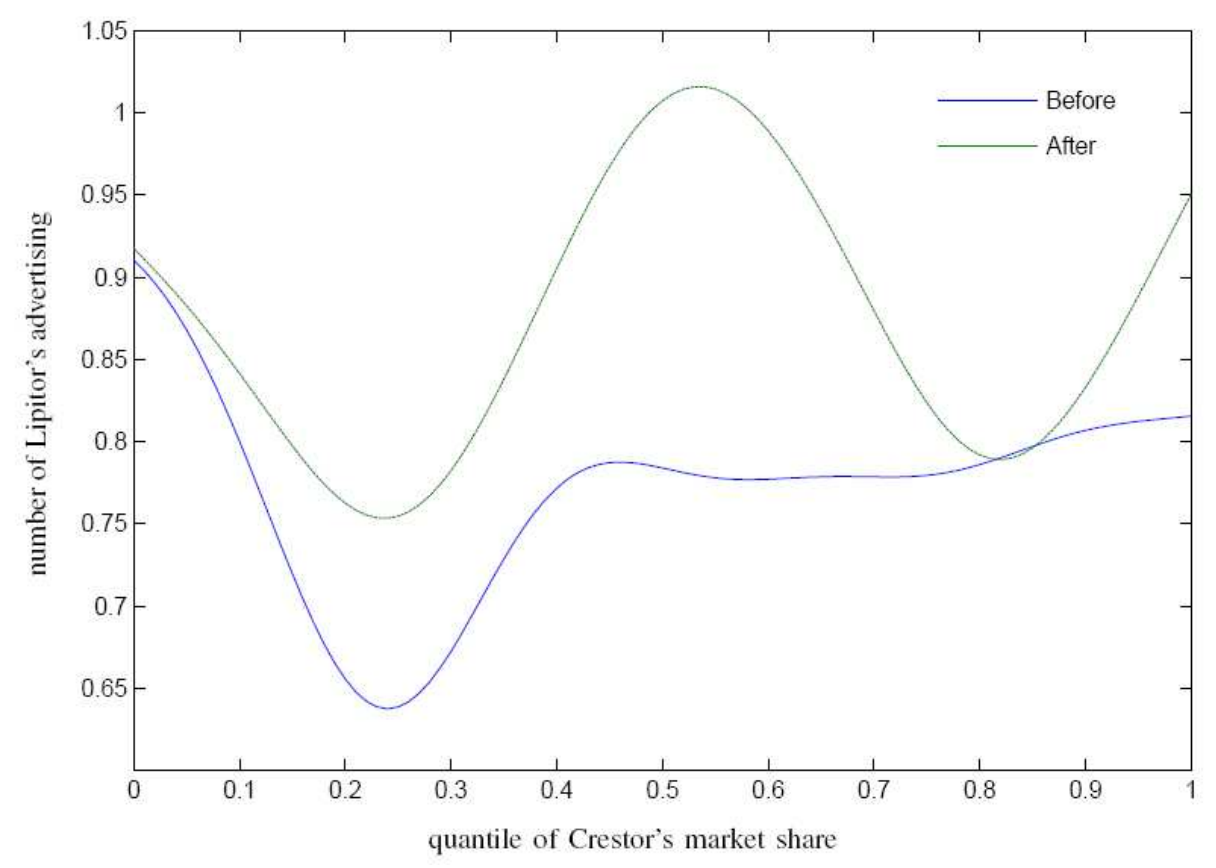

Figure 1: Lipitor's Advertising Before and After the News 


\section{The Model}

Let firm 1 be an incumbent and firm 2 be a new entrant. They compete in a market via demand-enhancing advertising when firm 2 suffers a demand-reducing shock. Firm 1 knows how the shock is going to affect the demand in virtue of its experience, while firm 2 does not know the exact effect of the shock. In the period immediately following the shock, firm 1 and firm 2 choose their advertising level simultaneously, then firm 2 decides to exit the market or not. If firm 2 exits, firm 1 becomes a monopolist in the following period, otherwise they compete under complete information and earn duopoly profits. Although firm 2 makes its exit choice without complete information regarding the effect of the shock, it might be able to infer the information by observing its first-period demand. Such inference procedure might in turn distort firm 1's advertising behavior in the first period.

The total demand in the market in each period is a constant, normalized to be 1 . Market price is fixed ${ }^{2}$ as $p$, and the unit cost of advertising for each firm is $c>0$. Firm 2's demand is given by $D\left(A_{1}, A_{2}, \gamma, \alpha\right) \in[0,1]$, and firm 1's demand is $1-D\left(A_{1}, A_{2}, \gamma, \alpha\right)$. Here $A_{1}, A_{2}$ are the advertising of firm 1 and firm 2, respectively; $\gamma$ catches the effect of the shock; $\alpha$ measures the market's loyalty to firm 2 before the shock, which is common knowledge to each firm. The function $D(\cdot)$ is continuously differentiable and satisfy the following assumptions:

$$
\text { A1. } D_{1}<0, D_{2}>0, D_{\alpha}>0, D_{\gamma}<0 \text {; }
$$

\footnotetext{
${ }^{2}$ There are several justifications for this assumption. Firstly, pharmaceutical companies compete mainly through advertising, and prices do not vary much across different brands. Secondly, prices are unlikely to adjust in the short period immediately following the shock, wherein there is strategic interaction between firm 1 and firm 2. Thirdly, this assumption allows us to abstract from other variables hence focus on firms' strategic advertising. Even if price is allowed to change, our basic findings shall not be altered. As a reference, see Bagwell and Ramey (1988) for an analysis on a signalling game with both advertising and pricing as strategic variables.
} 
A2. $D_{11}>0, D_{22}<0, D_{12}=0$;

A3. $D_{1 \gamma}<0, D_{1 \alpha}>0$.

A1 says that firm 2's demand is strictly increasing in $A_{2}$ and $\alpha$, strictly decreasing in $A_{1}$ and $\gamma$. A2 assumes that the own effect of advertising is diminishing, and the cross effect of advertising is zero. ${ }^{3}$ A3 guarantees that, the marginal effect of firm 1's advertising on firm 2's demand is strictly strengthened by a larger effect of the shock, and is strictly weakened by a stronger market loyalty to firm 2 .

We model firms 1 and 2's strategic interaction into a two-period game. In period one,

- stage 1: nature chooses the effect of the shock, which can take two values: a severe shock, represented by $\gamma_{H}$, or a mild shock, represented by $\gamma_{L}$, with $\gamma_{H}>\gamma_{L}$.

- stage 2: Firm 1 observes the value of $\gamma$ but firm 2 does not. Firm 2's prior probability assessment of the event that $\gamma=\gamma_{H}$ is $\rho \in(0,1)$.

- stage 3: firms 1 and 2 simultaneously choose their advertising investment, then demand/profits realize for each of them. Denote firm 2's period-one demand as $d$, which depends on two unobservable variables to firm $2, A_{1}$ and $\gamma$.

In period two,

- stage 1: firm 2 forms its posterior belief $\hat{\rho} \in[0,1]$ for $\gamma=\gamma_{H}$ based on the value of $d$.

- stage 2: firm 2 decides to exit or not, with $\xi=0(\xi=1)$ denoting exit (stay).

- stage 3: if firm 2 stays, it observes the value of $\gamma$ and compete with firm 1 under complete information. Each firm gets duopoly profits. Otherwise, firm 1 receives monopoly profits.

\footnotetext{
${ }^{3}$ We assume that there is no cross effect of firms' advertising in order to get analytical solution for the game. If this assumption is relaxed, firm 2's advertising will be responsive to the distortion in firm 1's advertising, which may vise versa enlarge the distortion in firm 1's advertising. Although this assumption abstracts out the strategic response of firm 2 to firm 1's signaling incentive, it is not the focus of this work.
} 
When firms 1 and 2 compete under complete information, their one period profits are

$$
\begin{aligned}
& \pi_{1}^{i}\left(A_{1}, A_{2}, \alpha\right)=p\left[1-D\left(A_{1}, A_{2}, \gamma_{i}, \alpha\right)\right]-c A_{1}-K, \\
& \pi_{2}^{i}\left(A_{1}, A_{2}, \alpha\right)=p D\left(A_{1}, A_{2}, \gamma_{i}, \alpha\right)-c A_{2}-K, \quad i=H, L
\end{aligned}
$$

Here $K>0$ is the fixed cost when a firm stays active in the market. ${ }^{4}$ Denote their duopoly profits as $\pi_{j D}^{i}$ at $\gamma=\gamma_{i}$ for firm $j$. Denote firm 1's monopoly profit as $\pi_{1 M}^{i}$ when firm 2 exits by ceasing advertising in that market. Assume $\pi_{1 M}^{H}>\pi_{1 D}^{H}, \pi_{1 M}^{L}>\pi_{1 D}^{L}$. I.e., whatever the type of the shock, firm 1 prefers firm 2 to exit.

If firms 1 and 2 compete only for one period under asymmetric information, their optimal advertising is denoted as normal competitive advertising, given by $\left(A_{1}^{H}, A_{1}^{L}, A_{2}^{E}\right)$ as

$$
\begin{aligned}
& A_{1}^{H} \equiv \arg \max _{A_{1}}\left[p\left(1-D\left(A_{1}, A_{2}^{E}, \gamma_{H}, \alpha\right)\right)-c A_{1}-K\right] \\
& A_{1}^{L} \equiv \arg \max _{A_{1}}\left[p\left(1-D\left(A_{1}, A_{2}^{E}, \gamma_{L}, \alpha\right)\right)-c A_{1}-K\right] \\
& A_{2}^{E} \equiv \arg \max _{A_{2}}\left\{p\left[\rho D\left(A_{1}^{H}, A_{2}, \gamma_{H}, \alpha\right)+(1-\rho) D\left(A_{1}^{L}, A_{2}, \gamma_{L}, \alpha\right)\right]-c A_{2}-K\right\} .
\end{aligned}
$$

It is easy to verify that $A_{1}^{H}>A_{1}^{L}$ (see the proof to Theorem 1). I.e., firm 1's normal competitive advertising is larger at a severe shock.

Now consider the two-period game. For a given strategy pair $\left(A_{1}, A_{2}\right)$ in period one and firm 2's exiting decision $\xi$ in period two, firm 1 and firm 2's discounted total profits are

$$
V^{i}\left(A_{1}, A_{2}, \xi\right)=\pi_{1}^{i}\left(A_{1}, A_{2}\right)+\left[\xi \pi_{1 D}^{i}+(1-\xi) \pi_{1 M}^{i}\right], \quad i=H, L
$$

for firm 1 and

$$
u^{i}\left(A_{1}, A_{2}, \xi\right)=\pi_{2}^{i}\left(A_{1}, A_{2}\right)+\xi \pi_{2 D}^{i}, \quad i=H, L
$$

\footnotetext{
${ }^{4}$ The fixed cost can be the fee of collecting market data, or the opportunity cost for a firm to continue its product promotion. $K>0$ gives firm 2 incentive to exit in order to avoid a negative profit.
} 
for firm 2. The discount factor is assumed to be 1 for simplicity. At the beginning of period one, firm 2's expected profit is

$$
U\left(A_{1}, A_{2}, \xi\right)=\rho u^{H}\left(A_{1}, A_{2}, \xi\right)+(1-\rho) u^{L}\left(A_{1}, A_{2}, \xi\right) .
$$

In period two, firm 2 chooses to stay only if it expects a non-negative duopoly profit in the future. The following lemma states that firm 2's duopoly profit is strictly increasing in $\alpha$.

Lemma $1 \frac{d \pi_{2 D}^{i}}{d \alpha}>0$ for $i=H, L$.

Proof: See the Appendix.

Define $\underline{\alpha} \equiv\left\{\alpha \mid \pi_{2 D}^{L}=0\right\}, \bar{\alpha} \equiv\left\{\alpha \mid \pi_{2 D}^{H}=0\right\}$. It follows that $\underline{\alpha}<\bar{\alpha}$ since $\pi_{2 D}^{H}<\pi_{2 D}^{L}{ }^{5}$ For $\alpha<\underline{\alpha}$, we have $\pi_{2 D}^{L}<0$ and $\pi_{2 D}^{H}<0$. Firm 2 will exit even under a mild shock. For $\alpha \geq \bar{\alpha}$, we have $\pi_{2 D}^{L}>0$ and $\pi_{2 D}^{H} \geq 0$. Firm 2 will stay even under a severe shock. In these two cases, firm 2's exit decision does not depend on the effect of the shock. Therefore, in period one, the incentive for firm 1 to signal firm 2 diminishes, and its optimal strategy is to maximize its period-one profit by advertising at the normal competitive level.

Theorem 1 If $\alpha<\underline{\alpha}$ or $\alpha \geq \bar{\alpha}$, the game has a unique perfect Bayesian Nash equilibrium, in which firms 1 and 2 advertise $\left(A_{1}^{i}, A_{2}^{E}\right)_{i=H, L}$ in period one as given in (2), then $\xi=0$ for $\alpha<\underline{\alpha}, \xi=1$ for $\alpha \geq \bar{\alpha}$.

\footnotetext{
${ }^{5}$ To see $\pi_{2 D}^{H}<\pi_{2 D}^{L}$, denote the equilibrium $A_{1}$ in period-two competition as $A_{1}^{*}(\gamma)$ at a given $\gamma$. By implicit function theorem,

$$
\frac{d A_{1}^{*}(\gamma)}{d \gamma}=-\frac{\operatorname{det} \frac{\partial(f 1, f 2)}{\partial\left(A_{2}, \gamma\right)}}{\operatorname{det} \frac{\partial(f 1, f 2)}{\partial\left(A_{2}, A_{1}\right)}}=-\frac{D_{1 \gamma}}{D_{11}}>0
$$
}

Here $f_{1}, f_{2}$ are first order conditions to profits in (1). By envelop theorem,

$$
\frac{d \pi_{2 D}}{d \gamma}=\frac{\partial \pi_{2 D}}{\partial A_{1}^{*}(\gamma)} \frac{d A_{1}^{*}(\gamma)}{d \gamma}+\frac{\partial \pi_{2 D}}{\partial \gamma}=p D_{1} \frac{d A_{1}^{*}(\gamma)}{d \gamma}+p D_{\gamma}<0 .
$$


Proof: Proof is straightforward hence omitted.

The interesting case is when $\alpha \in[\underline{\alpha}, \bar{\alpha})$, wherein $\pi_{2 D}^{L} \geq 0>\pi_{2 D}^{H}$ holds and firm 2 will exit only if the shock has a severe effect. While firm 2 tries to infer the value of $\gamma$ through its period-one demand, firm 1's incentive in advertising might be distorted. Our following analysis shall focus on the case when $\alpha \in[\underline{\alpha}, \bar{\alpha})$. We start from a formal introduction of the equilibrium concept.

\subsection{Equilibrium Concept}

The equilibrium concept we employ is Perfect Bayesian Nash equilibrium in pure strategy, refined by intuitive criterion in Cho and Kreps (1987). A Perfect Bayesian Nash equilibrium is a set of strategies $\left\{\left(\hat{A}_{1}^{i}, \hat{A}_{2}^{E}\right)_{i=H, L}, \hat{\xi}(d)\right\}$ and beliefs $\hat{\rho}(d)$, such that the following three conditions are satisfied.

Condition 1. Optimality for firm 1 . For $i=H, L$,

$$
\hat{A}_{1}^{i} \in \arg \max _{A_{1}} V^{i}\left(A_{1}, \hat{A}_{2}^{E}, \hat{\xi}(d)\right)
$$

Condition 2. Optimality for firm 2. For $i=H, L$,

$$
\hat{A}_{2}^{E} \in \arg \max _{A_{2}} U\left(\hat{A}_{1}^{i}, A_{2}, \hat{\xi}(d)\right)
$$

and for all $d, \hat{\xi}(d)=1$ if and only if

$$
\hat{\rho}(d) \pi_{2 D}^{H}+(1-\hat{\rho}(d)) \pi_{2 D}^{L} \geq 0
$$

Condition 3. Bayes' consistency of beliefs. If $D^{H}\left(\hat{A}_{1}^{H}, \hat{A}_{2}^{E}\right) \neq D^{L}\left(\hat{A}_{1}^{L}, \hat{A}_{2}^{E}\right)$, then $\hat{\rho}\left(D^{H}\left(\hat{A}_{1}^{H}, \hat{A}_{2}^{E}\right)\right)=1$ and $\hat{\rho}\left(D^{L}\left(\hat{A}_{1}^{L}, \hat{A}_{2}^{E}\right)\right)=0$. If $D^{H}\left(\hat{A}_{1}^{H}, \hat{A}_{2}^{E}\right)=D^{L}\left(\hat{A}_{1}^{L}, \hat{A}_{2}^{E}\right)$, then $\hat{\rho}\left(D^{H}\left(\hat{A}_{1}^{H}, \hat{A}_{2}^{E}\right)\right)=\hat{\rho}\left(D^{L}\left(\hat{A}_{1}^{L}, \hat{A}_{2}^{E}\right)\right)=\rho$.

If $d \notin\left\{D^{H}\left(\hat{A}_{1}^{H}, \hat{A}_{2}^{E}\right), D^{L}\left(\hat{A}_{1}^{L}, \hat{A}_{2}^{E}\right)\right\}$, any posterior belief is consistent. 
Condition 3 requires that firm 2's posterior beliefs about $\gamma$ be derived from Bayesian updating on its prior beliefs when the equilibrium advertising strategies are played. When firm 2 observes its period-one demand which does not occur under either type of the shock, any posterior belief $\hat{\rho}(d) \in[0,1]$ is fine. This mild condition gives rise to the multiplicity of both separating and pooling equilibria.

The "intuitive criteria" in Cho and Kreps (1987) is often invoked to refine the equilibrium set in signaling games. However, as Roberts (1986) points out, the idea is not directly applicable to a game where the recipient of the signal (firm 2) takes a simultaneous move with the sender of the signal (firm 1). ${ }^{6}$ Following Roberts (1986), an extension of the basic intuitive criteria is applied to the present game.

To refine the equilibrium set, we require that firm 2's beliefs after observing any particular period-one demand never put positive probability on $\gamma_{i}, i=H, L$ under which, given firm 2's period-one advertising, advertising of firm 1 which is necessary to generate such demand would represent deviation of firm 1 from the equilibrium strategy to a strategy dominated by the equilibrium choice. To be more explicit, fix an equilibrium at which advertising is $\left(\hat{A}_{1}^{i}, \hat{A}_{2}^{E}\right)_{i=H, L}$. The equilibrium demand of firm 2 in period one is given by $D^{i}\left(\hat{A}_{1}^{i}, \hat{A}_{2}^{E}\right)$. Now, consider an alternative pair $\tilde{A}_{1}^{i}$ such that $D^{i}\left(\tilde{A}_{1}^{i}, \hat{A}_{2}^{E}\right) \notin\left\{D^{H}\left(\hat{A}_{1}^{H}, \hat{A}_{2}^{E}\right), D^{L}\left(\hat{A}_{1}^{L}, \hat{A}_{2}^{E}\right)\right\}, i=H, L$. A deviation to $\tilde{A}_{1}^{i}$ is equilib-

\footnotetext{
${ }^{6}$ The game here is slightly different from standard signaling game. In a standard signaling game, the signal sender moves first and has its action (signal) observed by the signal receiver. Instead, in our game the signal sender (firm 1) moves simultaneously with the signal receiver (firm 2) in period one. Firm 2 does not observe firm 1's action (advertising). Instead, it observes its realized demand, which is an outcome of both firm 1's action and the effect of the shock. The simultaneity in the current game requires a tiny modification of the conventional wisdom when we refine the equilibrium set. With a little abuse of notation in our following analysis, we shall still use separating equilibrium for the equilibrium where firm 2 can tell the true effect of the shock, and pooling equilibrium for the equilibrium where firm 2 can not.
} 
rium dominated for firm 1 at $\gamma_{i}$ if

$$
V^{i}\left(\tilde{A}_{1}^{i}, \hat{A}_{2}^{E}, 0\right)<V^{i}\left(\hat{A}_{1}^{i}, \hat{A}_{2}^{E}, \hat{\xi}(d)\right)
$$

Thus when a deviation is equilibrium dominated at $\gamma_{i}$, the resulting profit of firm 1 is lower than its equilibrium profit irrespective of posterior beliefs. A sophisticated firm 1 at $\gamma_{i}$ should never choose such a deviation. Now we can define the notation of an intuitive equilibrium. An equilibrium strategy profile $\left\{\left(\hat{A}_{1}^{i}, \hat{A}_{2}^{E}\right)_{i=H, L}, \hat{\xi}(d)\right\}$ and beliefs $\hat{\rho}(d)$ is intuitive if and only if in addition to Conditions 1, 2 and 3, it satisfies

Condition 4. Elimination of equilibrium dominated strategies. That is, $\hat{\rho}\left(D^{i}\left(A_{1}, \hat{A}_{2}^{E}\right)\right)=$ $0(1)$ if $A_{1}$ is equilibrium dominated for firm 1 at $\gamma=\gamma_{H}\left(\gamma_{L}\right)$ but not at $\gamma=\gamma_{L}\left(\gamma_{H}\right)$.

\subsection{Separating Equilibria}

In a separating equilibrium, $D^{H}\left(\hat{A}_{1}^{H}, \hat{A}_{2}^{E}\right) \neq D^{L}\left(\hat{A}_{1}^{L}, \hat{A}_{2}^{E}\right)$. Observing demand in period one allows firm 2 to infer the true value of $\gamma$, and its exit decision will be based on full information. All separating equilibria involve firm 1 advertising its best response to firm 2's advertising when the shock is mild and, when the shock is severe, firm 1 advertises at a level that results in a value of $D^{H}\left(A_{1}, \hat{A}_{2}^{E}\right)$, such that firm 1 at a mild shock is not profitable to mimic, even though mimicry would induce exit. Although there generally is a continuum of separating equilibria, intuitive criterion refines separating equilibria to the least-cost one. In the intuitive equilibrium, firm 1's advertising when the shock is severe generates the demand of firm 2 , which makes firm 1 at the mild shock to be just indifferent between mimicking to induce exit and following its normal competitive strategy then getting the duopoly profits.

Under A2, the marginal effect of firm 2's advertising is invariant in firm 1's advertising. Therefore, firm 2's optimal advertising does not depend on its conjecture of firm 1's 
strategy, ${ }^{7}$ and $\hat{A}_{2}^{E}=A_{2}^{E}$ holds in equilibrium. For firm 1 , if $\gamma=\gamma_{L}$, it sets $\hat{A}_{1}^{L}=A_{1}^{L}$ since firm 2 infers complete information in a separating equilibrium and any $\hat{A}_{1}^{L} \neq A_{1}^{L}$ is strictly dominated by $A_{1}^{L}$ for firm 1 . Let us exploit the arbitrariness of off-equilibriumpath beliefs by setting $\hat{\rho}=1$ if $d=D^{H}\left(\hat{A}_{1}^{H}, A_{2}^{E}\right)$, and $\hat{\rho}=0$ otherwise, which means that firm 2 always stays unless its period-one demand is its equilibrium demand under a severe shock.

Given that firm 1 advertises $A_{1}$ at $\gamma_{H}$, if firm 1 wants to mimic at $\gamma_{L}$ by generating the same demand for firm 2, it advertises $x$ such that $D^{L}\left(x, A_{2}^{E}\right)=D^{H}\left(A_{1}, A_{2}^{E}\right)$. By the monotonicity of $D(\cdot)$, for each $A_{1}$ there exists a unique solution to firm 1's mimicry problem, given by $f\left(A_{1}\right) \equiv\left(D^{L}\right)^{-1}\left(D^{H}\left(A_{1}, A_{2}^{E}\right), A_{2}^{E}\right)$, such that $D^{L}\left(f\left(A_{1}\right), A_{2}^{E}\right)=$ $D^{H}\left(A_{1}, A_{2}^{E}\right)$. By $\mathrm{A} 1$ and $\mathrm{A} 3$, we have

$$
f\left(A_{1}\right)>A_{1}, \quad \frac{d f\left(A_{1}\right)}{d A_{1}}=-\frac{-D_{1}^{H}}{D_{1}^{L}}>1 .
$$

If $\hat{A}_{1}^{H}=A_{1}^{H}$, the following assumption says that, firm 1 at $\gamma_{L}$ will have incentive to mimic if doing so can convince firm 2 that $\gamma=\gamma_{H}$ :

$$
\text { A4. } \pi_{1}^{L}\left(f\left(A_{1}^{H}\right), A_{2}^{E}\right)+\pi_{1 M}^{L}>\pi_{1}^{L}\left(A_{1}^{L}, A_{2}^{E}\right)+\pi_{1 D}^{L} \text {. }
$$

A4 guarantees that, advertising $A_{1}^{H}$ at the severe shock by firm 1 can not induce firm 2 to set $\hat{\rho}=1$. Condition 1 requires that firm 1 at $\gamma_{L}$ should have no incentive to mimic therefore to induce firm 2's exit, implies

$$
\pi_{1}^{L}\left(f\left(\hat{A}_{1}^{H}\right), A_{2}^{E}\right) \leq \pi_{1}^{L}\left(A_{1}^{L}, A_{2}^{E}\right)-\left(\pi_{1 M}^{L}-\pi_{1 D}^{L}\right) \equiv \bar{\pi}_{1}^{L} .
$$

Thus $\hat{A}_{1}^{H}$ must give firm 2 a sufficiently low period-one demand under $\gamma=\gamma_{H}$, such that firm 1 at $\gamma_{L}$ will find it too costly to mimic. On the other side, Condition 1 requires

\footnotetext{
${ }^{7}$ The first order condition of firm 2's profit maximization under complete information is $p D_{2}^{i}-c=$ $0, i=H, L$. Let $A_{2}^{i}\left(A_{1}\right)$ be its solution. By implicit function theorem, $\frac{d A_{2}^{i}\left(A_{1}\right)}{d A_{1}}=-\frac{D_{12}^{i}}{D_{22}^{i}}=0$.
} 
firm 1 at $\gamma_{H}$ to prefer $\hat{A}_{1}^{H}$ to $A_{1}^{H}$, which means

$$
\pi_{1}^{H}\left(\hat{A}_{1}^{H}, A_{2}^{E}\right) \geq \pi_{1}^{H}\left(A_{1}^{H}, A_{2}^{E}\right)-\left(\pi_{1 M}^{H}-\pi_{1 D}^{H}\right) \equiv \underline{\pi}_{1}^{H} .
$$

As long as $\hat{A}_{1}^{H}$ satisfies both (I) and (II), Condition 1 is satisfied under our specification of beliefs, otherwise Condition 1 is violated for either $\gamma=\gamma_{H}$ or $\gamma=\gamma_{L}$ for any specification of beliefs. Condition 4 then reduces the set of $\hat{A}_{1}^{H}$ satisfying (I) and (II), when non-empty, to a unique one. The following theorem depicts the intuitive separating equilibrium, also gives the sufficient condition for its existence.

Theorem 2 Suppose $\alpha \in[\underline{\alpha}, \bar{\alpha})$. There exists at most one intuitive separating equilibrium, in which $\left\{\hat{A}_{1}^{H}=\bar{A}_{1}>A_{1}^{H}, \hat{A}_{1}^{L}=A_{1}^{L}, \hat{A}_{2}^{E}=A_{2}^{E}\right\}$. The sufficient condition for intuitive separating equilibrium to exist is $\pi_{1 M}^{H}-\pi_{1 D}^{H} \geq \pi_{1 M}^{L}-\pi_{1 D}^{L}$.

Proof: See the Appendix.

The proof contains multiple steps and proceeds as follows. It begins from showing the existence of $\bar{A}_{1}>A_{1}^{H}$, defined by $\pi_{1}^{L}\left(f\left(\bar{A}_{1}\right), A_{2}^{E}\right)=\bar{\pi}_{1}^{L}$, which uniquely maximizes $\pi_{1}^{H}\left(A_{1}, A_{2}^{E}\right)$ over all $A_{1}$ satisfying (I). Next, Condition 4 requires that $\pi_{1}^{L}\left(f\left(\hat{A}_{1}^{H}\right), A_{2}^{E}\right)=$ $\bar{\pi}_{1}^{L}$, otherwise there exists deviation which is equilibrium dominated for firm 1 at $\gamma_{L}$ but not at $\gamma_{H}$. It then follows that $\hat{A}_{1}^{H}$ must maximize $\pi_{1}^{H}\left(A_{1}, A_{2}^{E}\right)$ over (I), leading to $\hat{A}_{1}^{H}=\bar{A}_{1}$. Finally, the sufficient condition guarantees that $\bar{A}_{1}$ satisfies both (I) and (II).

The unique separating equilibrium thus incurs the least cost for firm 1 at $\gamma_{H}$ to signal. Firm 1 at $\gamma_{L}$ is just indifferent between mimicking by advertising $f\left(\bar{A}_{1}\right)$ and revealing the real value of $\gamma$ through one period optimal $A_{1}^{L}$.

\subsection{Pooling Equilibria}

In pooling equilibrium, $D^{H}\left(\hat{A}_{1}^{H}, \hat{A}_{2}^{E}\right)=D^{L}\left(\hat{A}_{1}^{L}, \hat{A}_{2}^{E}\right)$ and firm 2 learns nothing from observing its demand in period one. The following assumption is needed for a pooling equilibrium to exist: 


\section{A5. $\rho \pi_{2 D}^{H}+(1-\rho) \pi_{2 D}^{L}<0$.}

A5 says that firm 2 will exit according to its prior belief. If A5 is violated, firm 2 will always stay if it gets no new information on the value of $\gamma$. However, in this case there exists at least one severity level of the shock, either $\gamma_{H}$ or $\gamma_{L}$, at which firm 1 is profitable deviating to its one-period optimal advertising $\left(A_{1}^{H}\right.$ or $\left.A_{1}^{L}\right)$. This contradicts the definition of equilibrium. The following theorem gives the property of intuitive pooling equilibria.

Theorem 3 Suppose $\alpha \in[\underline{\alpha}, \bar{\alpha})$. When $\pi_{1 M}^{H}-\pi_{1 D}^{H} \geq \pi_{1 M}^{L}-\pi_{1 D}^{L}$ holds, there exist intuitive pooling equilibria. In any of them, $\left\{\hat{A}_{1}^{H} \in\left[A_{1}^{H}, \bar{A}_{1}\right], \hat{A}_{1}^{L}=f\left(\hat{A}_{1}^{H}\right), \hat{A}_{2}^{E}=A_{2}^{E}\right\}$.

Proof: See the Appendix.

It must be $\hat{A}_{1}^{H} \leq \bar{A}_{1}$ in pooling equilibrium for firm 1 at $\gamma_{L}$ to have incentive to mimic. The proof mainly contains two parts. The first part demonstrates the intuitiveness of the strategy in the theorem by contradiction. The second part rules out $A_{1}<A_{1}^{H}$ to support intuitive pooling equilibrium. The key property employed here is $\pi_{1}^{L}\left(f\left(A_{1}\right), A_{2}^{E}\right)-\pi_{1}^{L}\left(f\left(A_{1}^{\prime}\right), A_{2}^{E}\right)<\pi_{1}^{H}\left(A_{1}, A_{2}^{E}\right)-\pi_{1}^{H}\left(A_{1}^{\prime}, A_{2}^{E}\right)$ for $A_{1}>A_{1}^{\prime}$. That is, an increase in $A_{1}$ entails larger improvement in profits for firm 1 at $\gamma_{H}$ than at $\gamma_{L}$ when firm 1 at $\gamma_{L}$ is mimicking. If $\hat{A}_{1}^{H}<A_{1}^{H}$, then followed by the strict concavity of $\pi_{1}^{H}\left(A_{1}, A_{2}^{E}\right)$, there exists $A_{1}>A_{1}^{H}$ which is equilibrium dominated for firm 1 at $\gamma_{L}$ but not at $\gamma_{H}$.

In pooling equilibrium, firm 1 at $\gamma_{L}$ interferes firm 2's reference of the real value of $\gamma$, knowing that firm 2's optimal choice is to exit when no new information can be extracted. Although we have a continuum of pooling equilibria, there exists a unique Pareto-optimal one for each firm at either level of $\gamma$, given by $\left\{\hat{A}_{1}^{H}=A_{1}^{H}, \hat{A}_{1}^{L}=f\left(\hat{A}_{1}^{H}\right), \hat{A}_{2}^{E}=A_{2}^{E}\right\}$. At the Pareto optimal equilibrium, firm 1 at $\gamma_{L}$ upward-distorts its advertising, while firm 1 at $\gamma_{H}$ advertises at its normal competitive level. In all the other intuitive pooling equilibria, firm 1 at both $\gamma_{H}$ and $\gamma_{L}$ upward-distorts its advertising compared to the normal competitive level. 
To summarize, in equilibrium of the game, (1) if $\alpha$ is either too small or too large so that either $\pi_{2 D}^{L}<0$ or $\pi_{2 D}^{H} \geq 0$, firm 1 advertises according to its normal competitive strategy, $\left(A_{1}^{H}, A_{1}^{L}\right)$. There is no strategic element in firm 1's advertising; instead, (2) if $\alpha$ falls in the intermediate range so that $\pi_{2 D}^{L} \geq 0>\pi_{2 D}^{H}$, firm 1 upward-distorts its advertising either to reveal firm 2 its private information (separating equilibrium), or to disenable firm 2's reference of the real effect of the shock (pooling equilibrium).

\section{Relating the Theory to the Empirical Findings}

The theoretical predictions in Section 4 provide an explanation to the empirical findings in Chen and Tan (2009), as elaborated below.

When the news of Public Citizen's petition is released, Crestor needs to decide either to stay or to withdraw from the market, depending on the severity of the shock. As the bad news is not severe enough to force Crestor to fully withdraw from the Statin drug market immediately, the exit decision is more relevant at the physician level. Moreover, pharmaceutical firms mainly compete through advertising in the form of detailing. Therefore, we treat each physician as a market, and consider Crestor as having exited from a physician market if it stops detailing that physician. ${ }^{8}$

Since Lipitor is Crestor's major rival and the dominant brand, we focus on the potential strategic interaction between Lipitor and Crestor following the bad news. A critical assumption in our theoretical model is the existence of asymmetric information. We argue that, at the time of the withdrawal petition, Crestor was a new entrant, and it was Crestor's first time experiencing such bad news. Thus there was likely uncertainty in Crestor's belief regarding how badly the news was going to impact physicians' prescription behavior. On the other hand, Lipitor has interacted with physicians for a much longer period, and

\footnotetext{
${ }^{8}$ As long as Crestor's competitors prefer its stopping detailing a physician, the intrinsic incentive of predation persists under our definition.
} 
had witnessed a similar withdrawal petition on another drug, Baycol, and its subsequent withdrawal three years ago. ${ }^{9}$ Therefore, Lipitor was likely to possess superior information over Crestor regarding the effect of the bad news. In the period following the bad news, when Crestor remains active in the Statin drug market to infer the information regarding its future profitability, Lipitor could have incentive to signal Crestor an intrinsically bad market situation, with an eye towards Crestor's future withdrawal.

The theoretical work in Section 4 shows that, in equilibrium, Lipitor upward-distorts its advertising to physicians whose prior loyalty to Crestor lies in the intermediate range. Such prediction is consistent to the empirical findings of Chen and Tan (2009). Namely, Lipitor increases its advertising the most among physicians where Crestor has a prior median market share. ${ }^{10,11}$

One caveat exists in relating the theory to the empirical findings. In theory, the predicted upward-distortion in Lipitor's advertising is relative to Lipitor's one-period optimal advertising after the news (or in other words, its normal competitive advertising aimed at maximizing post-news profit without considering Crestor's future exit decision). However, Chen and Tan (2009) can only observe (therefore compare) Lipitor's advertising before and after the bad news. Thus there can be an alternative explanation to the empirical findings in Chen and Tan (2009). To be explicit, one could argue that, Lipitor's normal competitive advertising after the shock may possibly generate the same change in Lipitor's advertising patterns as found in Chen and Tan (2009).

To formally rule out this alternative explanation, let us assign $\gamma=\gamma_{o}$ to the demand

\footnotetext{
${ }^{9}$ For more details regarding the Statin drug market and the news event, see Section 3.

${ }^{10}$ In Chen and Tan (2009), Crestor's share in a physician's total prescription catches the loyalty level of that physician to Crestor.

${ }^{11}$ Among physicians where Crestor has either low or high prior market share, theoretical prediction is that Lipitor engages in its one-period optimal advertising, and there is an increase in its advertising from before to after the shock. This prediction is also consistent to the findings of Chen and Tan (2009).
} 
function before the shock occurs, with $\gamma_{o}<\gamma_{L}<\gamma_{H}$ to catch the fact that the news is demand-reducing for Crestor. Assume $\gamma_{o}$ to be common knowledge to both firms. Denote the normal competitive advertising of Lipitor (firm 1) and Crestor (firm 2) before the shock as $\left(A_{1}^{b}, A_{2}^{b}\right)$, and after the shock as $\left(A_{1}^{a}, A_{2}^{a}\right)$, abstracting out $\gamma=\gamma_{H}$ or $\gamma=$ $\gamma_{L}$. Denote $D^{b}=D\left(A_{1}^{b}, A_{2}^{b}, \gamma_{o}, \alpha\right), D^{a}=\left(A_{1}^{a}, A_{2}^{a}, \gamma, \alpha\right)$ the corresponding demand of Crestor before and after the shock.

Since $\frac{d\left(A_{1}^{a}-A_{1}^{b}\right)}{d \alpha}=\frac{D_{1 \alpha}^{b}}{D_{11}^{b}}-\frac{D_{1 \alpha}^{a}}{D_{11}^{a}}$, if $\frac{D_{1 \alpha}}{D_{11}}$ is monotonic in $\gamma,\left(A_{1}^{a}-A_{1}^{b}\right)$ never changes sign in $\alpha$. Whenever this is true, we can rule out the hypothesis that normal competitive advertising admits a non monotonic change in Lipitor's advertising with respect to $\alpha$. A sufficient condition for this to be true is

$$
\operatorname{sign}\left(D_{1 \alpha \gamma}\right)=-\operatorname{sign}\left(D_{11 \gamma}\right)
$$

Condition (4) requires that, a stronger market loyalty to Crestor and a larger $A_{1}$ should impose opposite impacts on the value of $D_{1 \gamma}$. Naturally we might expect $D_{1 \alpha \gamma}>0$ and $D_{11 \gamma}<0$. That is, the marginal impact of $\gamma$ on $D_{1}$ should be weakened by a stronger market loyalty towards Crestor, whereas strengthened by a more intensive advertising of Lipitor. Whenever (4) holds, a normal competitive response will always lead to a monotonic change in Lipitor's advertising before and after the news in the value of $\alpha$, contradicting the empirical findings of Chen and Tan (2009).

\section{Conclusion}

In this work, we theoretically model the advertising competition between an incumbent and a new entrant after a demand-reducing shock to the entrant, when the incumbent is privately informed as to the effect of the shock. A property of our model is that we allow for a heterogeneous loyalty to the entrant across different markets, therefore the incentive for the entrant to exit varies across markets. Our major finding is, in markets with median 
loyalty to the entrant such that exiting is optimal only under a severe shock, the incumbent upward-distorts advertising either to signal the entrant its private information or to interfere with the entrant's reference with the true effect of the shock, intended to induce the entrant to withdraw. In the rest of the markets, the incumbent advertises according to its one period optimal level in the absence of predatory incentive.

Our work thus offers a theoretical explanation to the empirical findings in Chen and Tan (2009). They treat each physician as a market and find that, after Crestor is petitioned to withdraw, its main competitor, Lipitor, demonstrates the largest increase in its advertising among physicians where Crestor has taken a median share in total prescriptions.

Our work proposes that a predatory intention could underlie Lipitor's post-shock advertising. The key insight is the existence of asymmetric information between Lipitor and Crestor regarding the severity level of the bad news. Having interacted with physicians for a much longer period and also witnessed a similar shock years ago, Lipitor shall have better information regarding how the shock is going to impact physicians' prescription behavior. As a result, among those physician markets where exiting becomes optimal for Crestor only under a several shock, Lipitor upward-distorts the demand-enhancing advertising to signal, with an eye towards Crestor's future withdrawal.

\section{Appendix}

Proof of Lemma 1. Maximizing profits in (1) gives the first order conditions for duopoly competition:

$$
\begin{array}{ll}
f 1: & -p D_{1}^{i}-c=0 \\
f 2: & p D_{2}^{i}-c=0 \quad i=H, L .
\end{array}
$$

Denote the solution as $\left(A_{1}^{i *}, A_{2}^{i *}\right)$. By implicit function theorem,

$$
\frac{d A_{1}^{i *}}{d \alpha}=-\frac{\operatorname{det} \frac{\partial(f 1, f 2)}{\partial\left(A_{2}, \alpha\right)}}{\operatorname{det} \frac{\partial(f 1, f 2)}{\partial\left(A_{2}, A_{1}\right)}}=-\frac{D_{1 \alpha}^{i}}{D_{11}^{i}}<0, \quad i=H, L .
$$


By envelop theorem,

$$
\frac{d \pi_{2 D}^{i}}{d \alpha}=\frac{\partial \pi_{2 D}^{i}}{\partial A_{1}^{i *}} \frac{d A_{1}^{i *}}{d \alpha}+\frac{\partial \pi_{2 D}^{i}}{\partial \alpha}=p D_{1}^{i} \frac{d A_{1}^{i *}}{d \alpha}+p D_{\alpha}^{i}>0, \quad i=H, L .
$$

complete the proof.

Proof of Theorem 2. The proof contains five steps. To simplify notation, let us suppress $A_{2}^{E}$ from $D^{i}\left(f\left(A_{1}\right), A_{2}^{E}\right), D^{i}\left(A_{1}, A_{2}^{E}\right), \pi_{1}^{i}\left(f\left(A_{1}\right), A_{2}^{E}\right)$ and $\pi_{1}^{i}\left(A_{1}, A_{2}^{E}\right), i=H, L$.

Step I. We show that $A_{1}^{H}>A_{1}^{L}$. By the definition of $A_{1}^{H}$ and $A_{1}^{L}$,

$$
\begin{aligned}
& p\left[1-D^{H}\left(A_{1}^{H}\right)\right]-c A_{1}^{H}>p\left[1-D^{H}\left(A_{1}^{L}\right)\right]-c A_{1}^{L} \\
& p\left[1-D^{L}\left(A_{1}^{L}\right)\right]-c A_{1}^{L}>p\left[1-D^{L}\left(A_{1}^{H}\right)\right]-c A_{1}^{H}
\end{aligned}
$$

Adding them gives $D^{L}\left(A_{1}^{H}\right)-D^{H}\left(A_{1}^{H}\right)>D^{L}\left(A_{1}^{L}\right)-D^{H}\left(A_{1}^{L}\right)$. By A3, $\left(D^{L}\left(A_{1}\right)-\right.$ $\left.D^{H}\left(A_{1}\right)\right)$ is strictly increasing in $A_{1}$. Thus $A_{1}^{H}>A_{1}^{L}$.

Step II. We show that there exists $\bar{A}_{1}>A_{1}^{H}$ such that $\pi_{1}^{L}\left(f\left(\bar{A}_{1}\right)\right)=\bar{\pi}_{1}^{L}$, which uniquely maximizes $\pi_{1}^{H}\left(A_{1}\right)$ over all $A_{1}$ which satisfies (I). First, notice that $\pi_{1}^{L}\left(f\left(A_{1}^{H}\right)\right)>$ $\bar{\pi}_{1}^{L}>\pi_{1}^{L}(f(\infty))$. By (3), $\pi_{1}^{L}\left(f\left(A_{1}\right)\right)$ is strictly decreasing in $A_{1}$ for $A_{1}>A_{1}^{H}>A_{1}^{L}$. By the continuity of $\pi_{1}^{L}\left(A_{1}\right)$, there exists a unique $\bar{A}_{1}>A_{1}^{H}$ such that $\pi_{1}^{L}\left(f\left(\bar{A}_{1}\right)\right)=\bar{\pi}_{1}^{L}$.

Second, we prove that $\bar{A}_{1}$ maximizes $\pi_{1}^{H}\left(A_{1}\right)$ over all $A_{1}$ which satisfies (I). by A4, $A_{1}^{H} \in\left(A_{1}^{L}, \bar{A}_{1}\right)$. Hence $\bar{A}_{1}$ is the maximizer to $\pi_{1}^{H}\left(A_{1}\right)$ over all $A_{1} \geq \bar{A}_{1}$ which satisfies (I). By the strict concavity of $\pi_{1}^{L}\left(A_{1}\right)$ and the fact $\pi_{1}^{L}\left(A_{1}^{L}\right)>\bar{\pi}_{1}^{L}$, there exists $\tilde{A}_{1}<A_{1}^{L}$ such that $\pi_{1}^{L}\left(f\left(\tilde{A}_{1}\right)\right)=\bar{\pi}_{1}^{L}$. Suppose $\tilde{A}_{1}>0$. Then $\pi_{1}^{H}\left(\tilde{A}_{1}\right)>\pi_{1}^{H}\left(A_{1}\right)$ for all $A_{1}<\tilde{A}_{1}$ which satisfies (I). We need to show that $\pi_{1}^{H}\left(\bar{A}_{1}\right)>\pi_{1}^{H}\left(\tilde{A}_{1}\right)$. Note that $\tilde{A}_{1}<A_{1}^{L}<$ $A_{1}^{H}<\bar{A}_{1}$. We have

$$
p\left[1-D^{L}\left(f\left(\tilde{A}_{1}\right)\right)\right]-c f\left(\tilde{A}_{1}\right)-p\left[1-D^{L}\left(f\left(\bar{A}_{1}\right)\right)\right]+c f\left(\bar{A}_{1}\right)=0 .
$$

Moreover, by (3), $\frac{d\left[f\left(A_{1}\right)-A_{1}\right]}{d A_{1}}=\frac{d f\left(A_{1}\right)}{d A_{1}}-1>0$, thus

$$
c\left(\tilde{A}_{1}-\bar{A}_{1}\right)>c\left(f\left(\tilde{A}_{1}\right)-f\left(\bar{A}_{1}\right)\right) .
$$


By (6) and (7),

$$
\begin{aligned}
& \pi_{1}^{H}\left(\bar{A}_{1}\right)-\pi_{1}^{H}\left(\tilde{A}_{1}\right)=p\left[1-D^{H}\left(\bar{A}_{1}\right)\right]-c \bar{A}_{1}-p\left[1-D^{H}\left(\tilde{A}_{1}\right)\right]+c \tilde{A}_{1} \\
& >p\left[D^{L}\left(f\left(\bar{A}_{1}\right)\right)-D^{H}\left(\bar{A}_{1}\right)\right]-p\left[D^{L}\left(f\left(\tilde{A}_{1}\right)\right)-D^{H}\left(\tilde{A}_{1}\right)\right]=0 .
\end{aligned}
$$

Step III. We argue that $\pi_{1}^{L}\left(f\left(\hat{A}_{1}^{H}\right)\right)=\bar{\pi}_{1}^{L}$ in a separating equilibrium. Suppose not. Since (I) must hold in equilibrium, we have $\pi_{1}^{L}\left(f\left(\hat{A}_{1}^{H}\right)\right)<\bar{\pi}_{1}^{L}$, i.e., $\hat{A}_{1}^{H}>\bar{A}_{1}$ or $\hat{A}_{1}^{H}<$ $\tilde{A}_{1}$. Consider a deviation from $\hat{A}_{1}^{H}$ to $\bar{A}_{1}+\epsilon$, with $\epsilon$ positive and arbitrarily close to zero. Since $\bar{A}_{1}+\epsilon$ satisfies (I), such a deviation is equilibrium dominated for firm 1 at $\gamma_{L}$. However, it is un-dominated by firm 1 at $\gamma_{H}$ as long as (II) is satisfied. Therefore, by Condition 4 , firm 2 should assign $\hat{\rho}=1$ upon observing such a deviation. Since $\bar{A}_{1}+\epsilon$ can be arbitrarily close to $\bar{A}_{1}$ and $\bar{A}_{1}$ is the unique maximizer of $\pi_{1}^{H}\left(A_{1}\right)$ over all $A_{1}$ satisfying (I), firm 1 has a profitable deviation at $\gamma_{H}$, which is a contradiction.

Step IV. We show that $\hat{A}_{1}^{H}=\bar{A}_{1}$. It follows from the argument in step III that $\hat{A}_{1}^{H}$ must maximize $\pi_{1}^{H}\left(A_{1}\right)$ over all $A_{1}$ satisfying (I), otherwise $\bar{A}_{1}+\epsilon$ is always a profitable deviation for firm 1 at $\gamma_{H}$. Hence $\hat{A}_{1}^{H}=\bar{A}_{1}$ in the intuitive separating equilibrium.

Step V. We show that $\pi_{1 M}^{H}-\pi_{1 D}^{H} \geq \pi_{1 M}^{L}-\pi_{1 D}^{L}$ is sufficient for the existence of intuitive separating equilibrium. It is immediate if $\bar{A}_{1}$ also satisfies (II). First, we show that there exists a unique $\overline{\bar{A}}_{1}>A_{1}^{H}$, such that $\pi_{1}^{H}\left(\overline{\bar{A}}_{1}\right)=\underline{\pi}_{1}^{H}$. Since $\pi_{1}^{H}(\infty)<\underline{\pi}_{1}^{H}<\pi_{1}^{H}\left(A_{1}^{H}\right)$, the existence of $\overline{\bar{A}}_{1}$ follows the continuity and monotonicity of $\pi_{1}^{H}\left(A_{1}\right)$ for $A_{1}>A_{1}^{H}$. Second, we show that $\bar{A}_{1}<\overline{\bar{A}}_{1}$. Since $\pi_{1}^{H}\left(A_{1}\right)-\pi_{1}^{L}\left(A_{1}\right)=p\left[D^{L}\left(A_{1}\right)-D^{H}\left(A_{1}\right)\right]$, we have

$$
\frac{d\left[\pi_{1}^{H}\left(A_{1}\right)-\pi_{1}^{L}\left(A_{1}\right)\right]}{d A_{1}}=p\left[D_{1}^{L}\left(A_{1}\right)-D_{1}^{H}\left(A_{1}\right)\right]>0
$$


due to A3. By (I) and (II),

$$
\begin{aligned}
& \pi_{1 M}^{H}-\pi_{1 D}^{H} \geq \pi_{1 M}^{L}-\pi_{1 D}^{L} \Rightarrow \pi_{1}^{H}\left(A_{1}^{H}\right)-\pi_{1}^{L}\left(A_{1}^{L}\right) \geq \pi_{1}^{H}\left(\overline{\bar{A}}_{1}\right)-\pi_{1}^{L}\left(f\left(\bar{A}_{1}\right)\right) \\
& \Rightarrow \pi_{1}^{H}\left(A_{1}^{H}\right)-\pi_{1}^{L}\left(A_{1}^{H}\right)>\pi_{1}^{H}\left(\overline{\bar{A}}_{1}\right)-\pi_{1}^{L}\left(f\left(\bar{A}_{1}\right)\right) \\
& \Rightarrow \pi_{1}^{H}\left(\overline{\bar{A}}_{1}\right)-\pi_{1}^{L}\left(\overline{\bar{A}}_{1}\right)>\pi_{1}^{H}\left(\overline{\bar{A}}_{1}\right)-\pi_{1}^{L}\left(f\left(\bar{A}_{1}\right)\right) \quad\left(\text { by }(8) \text { and } \overline{\bar{A}}_{1}>A_{1}^{H}\right) \\
& \Rightarrow f\left(\bar{A}_{1}\right)<\overline{\bar{A}}_{1} \quad\left(\text { since } f\left(\bar{A}_{1}\right)>A_{1}^{L}, \overline{\bar{A}}_{1}>A_{1}^{L}\right)
\end{aligned}
$$

By (3), $\bar{A}_{1}<\overline{\bar{A}}_{1}$ follows. Finally, since $\pi_{1}^{H}\left(A_{1}\right)$ is strictly decreasing in $A_{1}$ for $A_{1}>A_{1}^{H}$, it follows that $\pi_{1}^{H}\left(\bar{A}_{1}\right)>\underline{\pi}_{1}^{H}$. I.e., $\bar{A}_{1}$ satisfies (II).

Proof of Theorem 3. First, we show that the strategy stated in the theorem exists. It is clear since $\left[A_{1}^{H}, \bar{A}_{1}\right] \neq \varnothing$ following step II in the proof to Theorem 2 .

Second, we show the strategy stated in the theorem constitutes intuitive pooling equilibrium. Let us exploit the arbitrariness of off-equilibrium-path beliefs by setting $\hat{\rho}=\rho$ if $d=D^{H}\left(\hat{A}_{1}^{H}, A_{2}^{E}\right)$, and $\hat{\rho}=0$ otherwise, which means that firm 2 always updates its belief as the shock is mild with probability 1 unless its period-one demand is its equilibrium demand. By A2, $\hat{A}_{2}^{E}=A_{2}^{E}$. Given $A_{1}=\hat{A}_{1}^{H}$ at $\gamma_{H}$, firm 1 at $\gamma_{L}$ must have incentive to advertise $A_{1}=f\left(\hat{A}_{1}^{H}\right)$ in order to generate $D^{L}\left(A_{1}, A_{2}^{E}\right)=D^{H}\left(\hat{A}_{1}^{H}, A_{2}^{E}\right)$ for firm 2, as long as firm 2 exits upon observing such demand in period one. Thus $\left\{\hat{A}_{1}^{H}, \hat{A}_{1}^{L}, \hat{A}_{2}^{E}\right\}$ being in pooling equilibrium requires $\pi_{1}^{L}\left(f\left(\hat{A}_{1}^{H}\right)\right) \geq \bar{\pi}_{1}^{L}$. This is equivalent to requiring $\hat{A}_{1}^{H} \in\left[\tilde{A}_{1}, \bar{A}_{1}\right]$. Under $\pi_{1 M}^{H}-\pi_{1 D}^{H} \geq \pi_{1 M}^{L}-\pi_{1 D}^{L}$, we have $\bar{A}_{1}<\overline{\bar{A}}_{1}$. Thus $\hat{A}_{1}^{H} \in\left[A_{1}^{H}, \bar{A}_{1}\right]$ also satisfies (II). I.e., firm 1 at $\gamma_{H}$ is willing to advertise $A_{1} \in\left[A_{1}^{H}, \bar{A}_{1}\right]$ if only by doing so it can have firm 2 exit. Therefore, the strategy stated in the theorem together with the belief stated above satisfy Conditions 1, 2 and 3 .

Now we show that the strategy stated in the theorem satisfies Condition 4. Assume that there exists $A_{1}^{\prime} \neq \hat{A}_{1}^{H}$ such that

$$
\begin{gathered}
p\left[1-D^{H}\left(A_{1}^{\prime}\right)\right]-c A_{1}^{\prime}-p\left[1-D^{H}\left(\hat{A}_{1}^{H}\right)\right]+c \hat{A}_{1}^{H}>0 \\
p\left[1-D^{L}\left(f\left(\hat{A}_{1}^{H}\right)\right)\right]-c f\left(\hat{A}_{1}^{H}\right)-p\left[1-D^{L}\left(f\left(A_{1}^{\prime}\right)\right)\right]+c f\left(A_{1}^{\prime}\right)>0
\end{gathered}
$$


Noting that $D^{L}\left(f\left(\hat{A}_{1}^{H}\right)\right)=D^{H}\left(\hat{A}_{1}^{H}\right), D^{L}\left(f\left(A_{1}^{\prime}\right)\right)=D^{H}\left(A_{1}^{\prime}\right)$. Adding (9) and (10) gives

$$
c\left[f\left(A_{1}^{\prime}\right)-A_{1}^{\prime}\right]-c\left[f\left(\hat{A}_{1}^{H}\right)-\hat{A}_{1}^{H}\right]>0 .
$$

By (3), $f\left(A_{1}\right)-A_{1}$ is increasing in $A_{1}$. Thus $A_{1}^{\prime}>\hat{A}_{1}^{H} \geq A_{1}^{H}$, a contradiction to (9). Therefore, the strategy stated in the theorem satisfies Condition 4 and is intuitive.

Third, we show that there does not exist other intuitive pooling equilibrium. Firstly, $\hat{A}_{1}^{H}>\bar{A}_{1}$ is easy to rule out, since it is strictly dominated by $A_{1}^{L}$ for firm 1 at $\gamma_{L}$, therefore violates Condition 1 . Secondly, suppose $\hat{A}_{1}^{H}<A_{1}^{H}$. Then for any $A_{1}>\hat{A}_{1}^{H}$,

$$
\begin{aligned}
& \pi_{1}^{L}\left(f\left(A_{1}\right)\right)-\pi_{1}^{L}\left(f\left(\hat{A}_{1}^{H}\right)\right)=p D^{H}\left(\hat{A}_{1}^{H}\right)-p D^{H}\left(A_{1}\right)+c f\left(\hat{A}_{1}^{H}\right)-c f\left(A_{1}\right) \\
& <p D^{H}\left(\hat{A}_{1}^{H}\right)-p D^{H}\left(A_{1}\right)+c \hat{A}_{1}^{H}-c A_{1} \quad\left(\text { since } \frac{d\left(f\left(A_{1}\right)-A_{1}\right)}{d A_{1}}>0\right) \\
& =\pi_{1}^{H}\left(A_{1}\right)-\pi_{1}^{H}\left(\hat{A}_{1}^{H}\right) .
\end{aligned}
$$

Moreover, $\pi_{1}^{H}\left(A_{1}\right)$ is strictly decreasing in $A_{1}$ for $A_{1}>A_{1}^{H}$, and $\pi_{1}^{H}\left(A_{1}^{H}\right)>\pi_{1}^{H}\left(\hat{A}_{1}^{H}\right)>$ $\pi_{1}^{H}(\infty)$. By the continuity of $\pi_{1}^{H}\left(A_{1}\right)$ and $\pi_{1}^{L}\left(f\left(A_{1}\right)\right)$, there exists $A_{1}^{\prime}>A_{1}^{H}$ such that $\pi_{1}^{H}\left(A_{1}^{\prime}\right)=\pi_{1}^{H}\left(\hat{A}_{1}^{H}\right)$, whereas $\pi_{1}^{L}\left(f\left(A_{1}^{\prime}\right)\right)<\pi_{1}^{L}\left(f\left(\hat{A}_{1}^{H}\right)\right)$. Consider a deviation from $\hat{A}_{1}^{H}$ to $A_{1}^{\prime}-\epsilon$, with $\epsilon$ positive and arbitrarily close to zero, such that $\pi_{1}^{H}\left(A_{1}^{\prime}-\epsilon\right)>$ $\pi_{1}^{H}\left(\hat{A}_{1}^{H}\right), \pi_{1}^{L}\left(f\left(A_{1}^{\prime}-\epsilon\right)\right)<\pi_{1}^{L}\left(f\left(\hat{A}_{1}^{H}\right)\right)$. Intuitive beliefs then imply that $\hat{\rho}\left(D^{H}\left(A_{1}^{\prime}-\epsilon\right)\right)=$ 1 , and Condition 1 is violated for firm 1 at $\gamma_{H}$. Therefore, any $\hat{A}_{1}^{H}<A_{1}^{H}$ can not give intuitive pooling equilibrium strategies.

\section{References}

Albæk, S., Overgaard, P., 1992. "Upstream Pricing and Advertising Signal Downstream Demand.” Journal of Economics and Management Strategy, 1, 677-698.

Bagwell, K., Ramey, G., 1988. "Advertising and Limit Pricing." Rand Journal of Economics, 19, 59-71. 
Bagwell, K., Ramey, G., 1990. “Advertising and Pricing to Deter or Accommodate Entry When Demand is Unknown.” International Journal of Industrial Organization, 8, 93-113. Chen, Y., Tan W., 2009. "Strategic Advertising: Evidence in the Pharmaceutical Industry." working paper, SUNY-Stony Brook.

Cho, I., Kreps, D., 1987. “Signalling Games and Stable Equilibria.” Quarterly Journal of Economics, 102, 179-221.

Doraszelski, U., Markovich, S., 2007. "Advertising Dynamics and Competitive Advantage”. RAND Journal of Economics, 38, 557592.

Fudenberg, D., Tirole, J., 1984. “The Fat-cat Effect, The Puppy-dog Ploy, and The Lean and Hungry Look." American Economic Review, Papers and Proceedings, 74, 361-366.

Fudenberg, D., Tirole, J., 1986. “A ‘Signal-Jamming' Theory of Predation.” Rand Journal of Economics, 17, 366-376.

Hilke, J., Nelson, P., 1984. "Noisy Advertising and the Predation Rule in Antitrust Analysis." American Economic Review Proceedings, 74, 367-371.

Kreps, D., Wilson, R., 1982. "Reputation and Imperfect Information.” Journal of Economic Theory, 27, 253-279.

Linnemer, L., 1998. "Entry Deterrence, Product Quality: Price and Advertising as Signals." Journal of Economics and Management Strategy, 7, 615-645.

Mason, C., 1986. "Predation by Noisy Advertising." Review of Industrial Organization, 3, 78-92.

McGee, J., 1958. "Predatory Price Cutting: The Standard Oil (N.J.) Case." Journal of Law and Economics, 1, 137-169.

__, 1980. "Predatory Pricing Revisited." Journal of Law and Economics, 23, 443-459. Milgrom, P., Roberts, J., 1982. "Predation, Reputation, and Entry Deterrence.” Journal of Economic Theory, 27, 280-312. 
Milgrom, P., Roberts, J., 1986. "Price and Advertising Signals of Product Quality.” Journal of Political Economy, 94, 796-821.

Roberts, J., 1986. “A Signaling Model of Predatory Pricing." Oxford Economic Papers, $38,75-93$.

Saloner, G., 1987. "Predation, Mergers, and Incomplete Information." RAND Journal of Economics, 18, 165-186.

Schmalensee, R., 1983. "Advertising and Entry Deterrence: An Exploratory Model." Journal of Political Economy, 91, 636-653. 